\title{
HPVs detected in African Americans not represented in vaccines
}

\begin{abstract}
While cervical cancer continues to decrease, anal cancer incidence is on the rise with an estimated 8,080 new cases in the United States in 2016 and a 14\% mortality rate. In the past few decades, African American men have had the sharpest increase in incidence rates of anal cancer, as well as a shorter survival rate. Besides early detection of cervical cancer using pap smear, vaccines have also played a role in decrease of cervical cancer incidence. FDA recently approved a 9-valent vaccine which has the potential to increase prevention of ano-genital cancers from $70 \%$ to $90 \%$, but no research study has investigated whether the HPV types in 9-valent will target the HPV types which are most commonly associated in anal SCC with the African American (AA) cohort. In this pilot study, we analyzed the HPV genotype distribution in 24-HPV positive-anal lesions from the AA population using type-specific PCR. Overall, 17 different HPV types were detected where only HPV-16 and not 18 associated with anal SCC. HPV types 35 and 59 were associated with AIN2-3. Based on our study, it can be speculated that distribution of HPV genotypes may vary by race/ ethnicity. In general, it is our opinion that the new 9-valent vaccine might have limited value given that different races/ethnicities might have different genotypic distribution. For further validation, we suggest future investigations and clinical trials with inclusion of minority populations.
\end{abstract}

Keywords: HPV, vaccine, African American, cancer prevention, squamous cell carcinoma, anal cancer, cervical cancer
Volume 5 Issue I - 2016

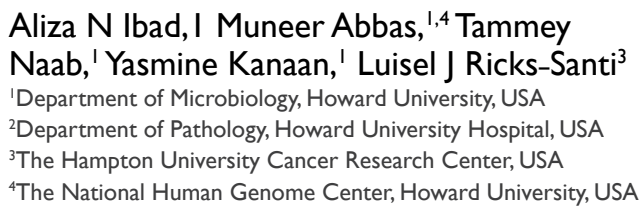

Correspondence: Luisel J Ricks-Santi, PhD, Director, Cancer Research Center, Hampton University, 100 E Queen Street, Hampton,VA 23668, USA, Tel 757-727-563I, Email M_Abbas@Howard.edu

Received: May 19, 2016 | Published: June 15, 2016
Abbreviations: AA, African American; AIN, anal intraepithelial neoplasia; CIN, cervical intraepithelial neoplasia; FFPE, formalin fixed paraffin embedded; HPV, human papilloma virus; HR, high risk; ICC, invasive cervical cancer; LR, low risk; SCC, squamous cell carcinoma

\section{Introduction}

When compared to cervical cancer, anal cancer is an uncommon malignancy, but its incidence is on the rise. The Surveillance, Epidemiology, and End Results (SEER) report predicts an estimated 8,080 new cases of anal cancer in United States (US). Even though it is just $0.5 \%$ of all new cancer cases in the US, the mortality rate is estimated at $14 \%$, which is comparable to both prostate and breast cancer mortality rates. In the past three decades, incidence rates of anal cancer have significantly increased with the highest increase in African American (AA) men. ${ }^{1}$ AA men also had a lower survival rate from the disease; the five-year survival rate for AA men with early stage disease was 62 percent as compared to 79 percent for Caucasian men with localized cancer. ${ }^{2}$ Ninety-five percent $(95 \%)$ of anal squamous cell carcinomas (SCC) are attributed to human papillomavirus (HPV) which is also the primary cause of cervical cancers. ${ }^{1,3}$ While cervical cancer continues to decrease which could be attributed to a myriad of factors-including early detection made possible through pap smears and efficacy of vaccines - anal cancer incidence is on the rise.

Since 2006, the Federal Drug Administration (FDA) has approved three prophylactic vaccines to prevent HPV infection which can also lead to ano-genital cancers: Cervarix, Gardasil, and Gardasil 9, all three of which prevent infections with HPV high-risk types 16 and 18. Gardasil (quadrivalent) also prevents infection with HPV low-risk types 6 and 11, which cause 90 percent of genital warts. ${ }^{4}$ Just recently, the FDA approved a brand new vaccine, Gardasil 9, which is supposed to prevent infection with the same four HPV types plus an additional five high-risk (HR) HPV types (31, 33, 45, 52, and 58), and is therefore called a nonavalent, or 9-valent, vaccine. In recent years, investigations have suggested that AA women are half as likely to get infected with HR-HPV 16 and 18, but instead have a higher chance of infection due to HR-HPV 35, 45, 58 and 68 when looked at in cervical cancer. In addition, the mortality rate is two times higher in women of African ancestry. ${ }^{5-7}$ Based on the key biological similarity between Anal Intraepithelial Neoplasia (AIN) and Cervical Intraepithelial Neoplasia (CIN), which depends on the causal relation between the oncogenic or HR-HPV and the development of SCC and the cervix (ICC), ${ }^{8}$ one would question not only the efficacy of these vaccines on prevention of cervical cancer in AA women, but also on prevention of anal cancer in the AA population as a whole.

Our team at the National Human Genome Center and the Howard University Cancer Center conducted a small study to analyze the HPV genotype distribution in $24 \mathrm{HPV}$ positive anal lesions (consisting of AIN1-3 and SCC) of an AA cohort following IRB approval. Formalin fixed paraffin embedded (FFPE) anal tissue biopsies were obtained from Howard University Hospital, Department of Pathology. The average age of patients was 44 (range $23-61$ ), and $80 \%$ of patients were males. FFPE blocks were sectioned on microtome and deparaffinized using Xylene. DNA was extracted using Qiagen FFPE DNA Isolation Kit and quantified using Qubit Fluorometric Quantitation. Extracted DNA with a minimum concentration of $20 \mathrm{ng} / \mu \mathrm{l}$ was used for type-specific PCR. DNA sequence files for HPV types $6,11,16,18$, $26,31,32,33,34,35,39,45,51,52,53,56,58,59,61,68,73$ and 82 were obtained from PaVE:Papilloma virus genome database. Primers were designed using Primer Quest Tool with the non-conservative E6 gene for each HPV type, and unique specificity was confirmed by BLAST analysis. The 22 type-specific PCRs were performed in parallel as described by Lin et al. ${ }^{9}$ Positive bands were visualized on an ethidium bromide stained agarose gel. 
The sensitive type-specific PCR approach lead to all 24 samples testing positive for at least one HPV type. HR-HPV 16 was most commonly associated with anal SCC while HR-HPV 18 was not found in any of the SCC lesions. AIN lesions1-3 and specifically moderate to high grade AIN were also associated with HPV types 16, 32, 35, 51, 58,59 , and 68 . In spite of a small sample size, our results represent a strong correlation to two studies performed by Vidal et al. \& Hariri et al..$^{5,10}$ in 2012 which also reported HPV types 35 and 58 as the most commonly found genotypes in CIN1-3 and ICC among AA women, instead of HPV-18.

\section{Conclusion}

To this day, there is a dearth of studies that have investigated the efficacy of HPV vaccines on anal cancer and none have focused on ethnically diverse populations. Even though HPVs 58 and 68 are covered by the nonavalent vaccine, HPVs 32, 35, 51 and 59 are not, which may give the vaccine limited value in preventing anal SCC and more importantly in preventing premalignant (AIN) lesions in the AA population. While our findings strongly support the results from both Vidal et al. \& Hariri et al. ${ }^{5,10}$ it is important to note that our sample size was small. Still, one may speculate that HPV genotype might differ across different racial/ethnic backgrounds given our data which demonstrates the heterogeneity of HPV infections. We surmise that this HPV genotype distribution can provide one possible explanation for higher incidence and lower survival rate of AA men affected by HPV infection. This difference in genotype distribution is clearly visible in premalignant lesions, and although HR-HPV 16 is the oncogenic type most commonly associated with anal SCC, one needs to keep in mind that premalignant lesions (AIN1-3) are generally a precursor of malignant SCC. ${ }^{11}$ If premalignant lesions of minority population are infected by HPV types that are not targeted by the vaccine, we need to reconsider the clinical validity and utility of current vaccines to ensure that both precancerous and cancerous anal lesions are targeted. We hope the results from this pilot study would lead to further investigations into HPV genotypic distribution of minority populations and the efficacy of current vaccines so that we are able to close the racial disparities gap in HPV-induced cancers.

\section{Acknowledgments}

None.

\section{Conflicts of interest}

Authors declare that there is no conflict of interest.

\section{References}

1. Daling JR, Madeleine MM, Johnson LG, et al. Human papillomavirus, smoking, and sexual practices in the etiology of anal cancer. Cancer. 2004;101(2):270-280.

2. Johnson LG, Madeleine MM, Newcomer LM, et al. Anal cancer incidence and survival:the surveillance, epidemiology, and end results experience, 1973-2000. Cancer. 2004;101(2):281-288.

3. Palefsky JM. Human papillomavirus infection and anogenital neoplasia in human immunodeficiency virus-positive men and women. Journal of the National Cancer Institute. Monographs. 1998;23:15-20.

4. Koutsky LA, Ault KA, Wheeler CM, et al. A controlled trial of a human papillomavirus type 16 vaccine. NEnglJMed. 2002;347(21):1645-1651.

5. Hariri S, Unger ER, Powell SE, et al. Human papillomavirus genotypes in high-grade cervical lesions in the United States. $J$ Infect Dis. 2012;206(12):1878-1886.

6. Niccolai LM, Russ C, Julian PJ, et al. Individual and geographic disparities in human papillomavirus types 16/18 in high-grade cervical lesions:Associations with race, ethnicity, and poverty. Cancer. 2013;119(16):3052-3058.

7. Susan K, Murphy CH. What are the Implications of Distinct HPV Genotypes in Women of Different Ethnic/Racial Ancestry? Journal of Vaccines \& Vaccination. 2014;05(03):5-7.

8. Darragh TM, Winkler B. Anal cancer and cervical cancer screening:Key differences. Cancer Cytopathol. 2011;119(1):5-19.

9. Lin CY, Chao A, Yang YC, et al. Human papillomavirus typing with a polymerase chain reaction-based genotyping array compared with type-specific PCR. J Clin Virol. 2008;42(4):361-367.

10. Vidal AC, Smith JS, Valea F, et al. HPV genotypes and cervical intraepithelial neoplasia in a multiethnic cohort in the southeastern USA. Cancer Causes Control. 2014;25(8):1055-1062.

11. Poggio JL. Premalignant lesions of the anal canal and squamous cell carcinoma of the anal canal. Clin Colon Rectal Surg. 2011;24(3):177-192. 